\title{
CORRELATION BETWEEN GALACTIC NON-THERMAL CONTINUUM EMISSION AND HI-COLUMN DENSITY
}

\author{
K. BEUERMANN and Y.G. KIM \\ Institut für Astronomie und Astrophysik \\ Technische Universität Berlin, PN 8-1 \\ Hardenbergstr. 36 \\ D-1000 Berlin 21
}

The distribution of magnetic field strengths in the interstellar gas is an important datum which relates to the formation of dense cloud complexes as well as to the distribution and propagation of cosmic rays. Brown and Chang (1983) found the brightness temperature of the radio continuum emission to be correlated with the HI column density $\mathrm{N}_{\mathrm{H}}$ and deduced a field strength which increases with particle density as $\langle\mathrm{H}\rangle \sim\langle\mathrm{n}\rangle \mathbf{0 . 4 4}$. Troland and Heiles (1986) critized their approach and pointed out that Zeeman measurements suggest no increase in field strength for particle densities $\mathrm{n} \leq 100 \mathrm{H}$-atoms $\mathrm{cm}^{-3}$.

We have reinvestigated this question using an approach which differs from that of Brown and Chang. Beuermann, Kanbach, and Berkhuijsen (1985) noted that a fraction of the non-thermal continuum emission at $408 \mathrm{MHz}$ (Haslam et al., 1981) is, in fact, connected with the HI layer. This component can only be isolated, however, in regions which are sufficiently free of radiation from HII regions and supernova remnants. Suitable regions with size ranging from 30 to 250 square degrees were selected in the longitude and latitude ranges $1=62^{\circ}$ to $70^{\circ}, 1=145^{\circ}$ to $250^{\circ}$, and $b=-4^{\circ}$ to $+4^{\circ}$. For faint extended and point sources we applied appropriate corrections. Subsections affected by strong sources were excluded. For these regions, our new findings can be summarized as follows:

(1) A correlation of the Form $T_{b}(408 \mathrm{MHz})=A+\mathrm{BN}_{\mathrm{HI}}$ is found everywhere along the plane. The underlying (diffuse) component $A$ is typically stronger than the contribution $\mathrm{BN}_{\mathrm{HI}}$ from the $\mathrm{HI}$ gas. The quantity $A$ increases systematically along the galactic plane. $B$ is in the range $(1.0-2.0) 10^{-21} \mathrm{~K} \mathrm{H}$-atoms ${ }^{-1} \mathrm{~cm}^{2}$ with a mean value of $(1.4 \pm 0.3) 10^{-21}$ (Fig. 1).

(2) Given the synchrotron emissivity of the near-solar electron spectrum at $408 \mathrm{MHz}, \varepsilon=(0.34 \pm 0.09) \mathrm{H}_{1}^{1.8} \mathrm{~K} \mathrm{kpc}^{-1}$ and assuming the density of the relativistic electrons to be constant, we obtain for the mean line-of-sight values of the cloud component

$$
\left\langle H_{\perp}^{1.8}\right\rangle=(12.7 \pm 4.2)\langle n\rangle
$$

with $H_{\perp}$ in $\mu G$ and $n$ in $H_{-}^{-a t o m s ~} \mathrm{~cm}^{-3}$. 
(3) This result demonstrates that HI clouds have field strengths which exceed those of the surrounding intercloud medium. The field strength as a function of density in $\mathrm{HI}$ clouds cannot be extracted from this result and, hence, a comparison with $\mathrm{H}-\mathrm{n}$ relations predicted for collapsing clouds is not possible.

Our result indicates that there is a component of the galactic non-thermal synchrotron emission which originates from HI clouds. Because of its small emissivity it is not easy, however, to sepatate it from the large-scale diffuse background (and foreground) component which varies with longitude and latitude. The brightness temperature of the cloud component amounts on the average to only $\sim \mathrm{K}$ towards the anticenter and $20 \mathrm{~K}$ towards the galactic center. Its total contribution to the monochromatic power of the Galaxis at $408 \mathrm{MHz}$ is only 3\% (Beuermann et al., 1985).

\section{$\underline{\text { References }}$}

Beuermann, K., Kanbach, G., Berkhuijsen, E.M.: 1985, Astron. Astrophys. 153, 17 Brown, R.L., Chang, C.-A.: 1983, Astrophys. J. 264, 134

Haslam, C.G.T., Salter, C.J., Stoffel, H., Wilson, W.E.: 1981, Astron. Astrophy. Suppl. 47, 1

Troland, T.H., Heiles, C.: 1986, Astrophys. J. 301, 339

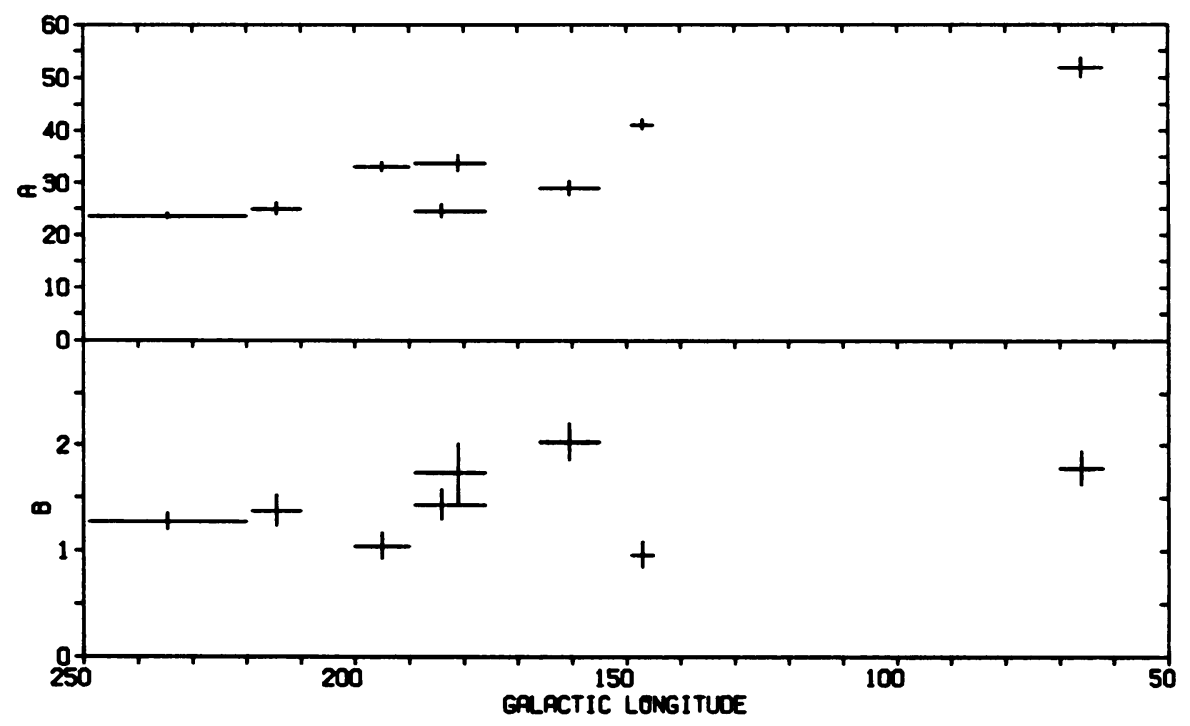

Figure 1. Result of the correlation study between brightness temperature $T_{b}$ at $408 \mathrm{MHz}$ and the integrated $\mathrm{HI}$ column density $\mathrm{N}_{\mathrm{HI}}$. The coefficients of the linear fit $T_{b}=A+B N_{H I}$ are given for individual selected regions near the galactic plane. $A$ is in $\mathrm{K}$ and $B$ in units of $10^{-21} \mathrm{~K}\left(\mathrm{H} \text {-atoms } \mathrm{cm}^{-2}\right)^{-1}$. 